\title{
Different copies of SENSITIVITY TO RED LIGHT REDUCED 1 show strong subfunctionalization in Brassica napus
}

\author{
Sarah Schiess| ${ }^{2+}$, Natalie Williams ${ }^{1+}$, Pascal Specht ${ }^{2}$, Dorothee Staiger ${ }^{1}$ and Mikael Johansson ${ }^{1 *}$ (D)
}

\begin{abstract}
Background: Correct timing of flowering is critical for plants to produce enough viable offspring. In Arabidopsis thaliana (Arabidopsis), flowering time is regulated by an intricate network of molecular signaling pathways. Arabidopsis srr1-1 mutants lacking SENSITIVITY TO RED LIGHT REDUCED 1 (SRR1) expression flower early, particularly under short day (SD) conditions (1). SRR1 ensures that plants do not flower prematurely in such non-inductive conditions by controlling repression of the key florigen FT. Here, we have examined the role of SRR1 in the closely related crop species Brassica napus.

Results: Arabidopsis SRR1 has five homologs in Brassica napus. They can be divided into two groups, where the A02 and C02 copies show high similarity to AtSRR1 on the protein level. The other group, including the A03, A10 and C09 copies all carry a larger deletion in the amino acid sequence. Three of the homologs are expressed at detectable levels: A02, C02 and C09. Notably, the gene copies show a differential expression pattern between spring and winter type accessions of $B$. napus. When the three expressed gene copies were introduced into the srr $1-1$ background, only A02 and CO2 were able to complement the srr1-1 early flowering phenotype, while CO9 could not. Transcriptional analysis of known SRR1 targets in Bna.SRR1-transformed lines showed that CYCLING DOF FACTOR 1 (CDF1) expression is key for flowering time control via SRR1.

Conclusions: We observed subfunctionalization of the B. napus SRR1 gene copies, with differential expression between early and late flowering accessions of some Bna.SRR1 copies. This suggests involvement of Bna.SRR1 in regulation of seasonal flowering in B. napus. The C09 gene copy was unable to complement srr1-1 plants, but is highly expressed in B. napus, suggesting specialization of a particular function. Furthermore, the C09 protein carries a deletion which may pinpoint a key region of the SRR1 protein potentially important for its molecular function. This is important evidence of functional domain annotation in the highly conserved but unique SRR1 amino acid sequence.
\end{abstract}

Keywords: Arabidopsis, Brassica napus, Flowering, Cross-species functionality, Subfunctionalization

\section{Background}

Plants need to synchronize their reproductive activity to the optimal growth season, to ensure maximal reproductive output. Consequently, onset of flowering is tightly controlled by a network of signals originating from developmental, as well as environmental signaling pathways [2-4]. After reaching a critical developmental age, plants will respond to favorable

\footnotetext{
* Correspondence: mikael.johansson@uni-bielefeld.de

${ }^{\dagger}$ Sarah Schiessl and Natalie Williams contributed equally to this work.

${ }^{1}$ RNA Biology and Molecular Physiology, Faculty for Biology, Bielefeld

University, Universitaetsstrasse 25, 33615 Bielefeld, Germany

Full list of author information is available at the end of the article
}

environmental stimuli and flowering will be initiated [5]. In long day (LD) plants, flowering is promoted in spring and summer when the days are longer than the nights. Day length is measured by the inner circadian clock that maintains a ca 24-h cyclic rhythm of gene and protein expression of clock components that in turn regulate downstream processes. When light coincides with the expression of components of the so-called photoperiodic pathway of flower induction, expression of "florigen" FLOWERING LOCUS T $(F T)$ is promoted in the leaves [6, 7]. FT protein then travels through the vasculature to the shoot apex where flower formation is initiated [8-10]. CONSTANS (CO) is a key signal

(c) The Author(s). 2019 Open Access This article is distributed under the terms of the Creative Commons Attribution 4.0 International License (http://creativecommons.org/licenses/by/4.0/), which permits unrestricted use, distribution, and 
integrator for photoperiodic flowering. Its transcription is controlled by the circadian clock through the GIGANTEA (GI) clock component that interacts with FLAVIN BINDING, KELCH REPEAT, F-Box 1 (FKF1) in coincidence with light. FKF1 then represses the activity of CDF transcription factors, which have a repressive role on $\mathrm{CO}$ expression [11-13]. This allows accumulation of $\mathrm{CO}$ transcript in the afternoon and $\mathrm{CO}$ protein expression. $\mathrm{CO}$ in turn promotes expression of $F T$ by binding to its promoter and thus initiating flowering. Transcription of $F T$ is also tightly regulated by both promotive and repressive elements that integrate signals from various environmental and developmental signaling pathways [2]. An important $F T$ repressor in this transcriptional landscape is the MADS box transcription factor FLOWERING LOCUS C (FLC), which has an important role as a repressor of flowering in unfavorable conditions, as its expression level is reduced by extended periods of cold $[14,15]$.

The main genetic factors of the flowering time regulation network have been conserved throughout Brassicaceae, as revealed by genome sequencing in recent years [16-20]. This conservation indicates that their function might be similar as in the model species Arabidopsis. Additionally, many quantitative trait loci (QTL) studies and genome-wide association studies (GWAS) for flowering time have found homologs of Arabidopsis flowering time genes in the confidence intervals of associated markers [21-29]. However, the most important crop plants from the Brassicaceae come from the genus Brassica, including important vegetable species like cabbage, cauliflower (Brassica oleracea), Chinese cabbage (Brassica rapa), but also the important oilseed crop rapeseed (Brassica napus). Brassica species share a whole-genome triplication, and $B$. napus arose from a recent interspecific hybridization between $B$. rapa (A subgenome donor) and $B$. oleracea ( $C$ subgenome donor), expanding the theoretically expected copy number of Arabidopsis homologs in allotetraploid B. napus to 6 (Brassica triplication $\times 3$, hybridization $\times 2$ ) $[30,31]$. After polyploidization, many different processes like homologous recombination and the action of transposable elements led to a strong genome reorganization. Together with selective processes, this reorganization individually changed the specific gene copy numbers, now varying between 1 and 12, and possibly varying between individuals $[16,32,33]$. In the course of evolution, single copies might evolve differently and give rise to new expression patterns or functions through a process called subfunctionalization [34]. The degree of subfunctionalization is gene specific. Subfunctionalization has played an important role in evolution of flowering time control $[35,36]$.

SENSITIVITY TO RED LIGHT REDUCED (SRR1) is essential for repression of flowering in non-inductive photoperiods in Arabidopsis [1]. Mutant srr1-1 plants flower particularly early under SD conditions and show a reduced sensitivity to the lengthening of the photoperiod. SRR1 acts to promote the expression of several direct repressors of $F T$, including $C D F 1$, the TEMPRANILLO (TEM) transcription factors that are also involved in gibberellic acid biosynthesis and $F L C$, ensuring that flowering is prevented in non-inductive conditions. In addition, SRR1 has roles in setting the correct pace of the circadian clock and in mediating red light signaling [37]. SRR1 was also found to be important for control of flowering time in natural conditions, together with many genes closely associated to the circadian clock in a combined genome-wide association (GWAS) and linkage mapping study in Arabidopsis [38]. The protein structure of SRR1 is unknown and it does not contain any known protein motifs, although it is highly conserved between species, with homologs present in yeast and mammals [37, 39]. In Brassica rapa, a quantitative trait loci (QTL) study combining whole genome transcript variation with flowering time QTLs, identified the $B r S R R 1$ ortholog as a candidate associated with flowering and the expression of BrFT [40]. Furthermore, the Bna.SRR1.A02 copy has recently been identified as one of the candidates genes responsible for the morphotypic split between biannual and annual forms in B. napus [41]. This suggests that the role for SRR1 in flowering time control may be conserved among Brassicaceae.

B. napus carries 5 copies of Bna.SRR1 located on chromosomes A02, A03, A10, CO2 and C09. It is unclear if all of them have maintained the original function or if they have undergone subfunctionalization processes. Here, we examine the functionality of the Bna.SRR1 copies by expression analysis in $B$. napus and complementation of Arabidopsis srr1-1 mutants. We show that two groups of different gene structures have evolved and that only some Bna.SRR1 gene copies are functional in Arabidopsis. This indicates a strong subfunctionalization of Bna.SRR1 and provides new information about SRR1 function.

\section{Results}

\section{Phylogeny of SRR1 in Brassicaceae}

We searched 13 sequenced Brassicaceae species for homologs of A. thaliana SRR1. Copies of SRR1 were found in all 13 species (Fig. 1a). Most of them (8 out of 13, A. thaliana, A. lyrata, Capsella rubella, Thelungiella salsunginea, Thelungiella halophila, Aethionema arabicum, Leavenworthia alabamicum, Schrenkiella parvula) carried only one copy of SRR1, whereas $B$. rapa and B.oleracea each carried two copies, Camelina sativa carried three copies, Sisymbrium irio four copies and $B$. napus five copies. Thus, B. napus carries one copy more than expected from its progenitor species. 
(a)

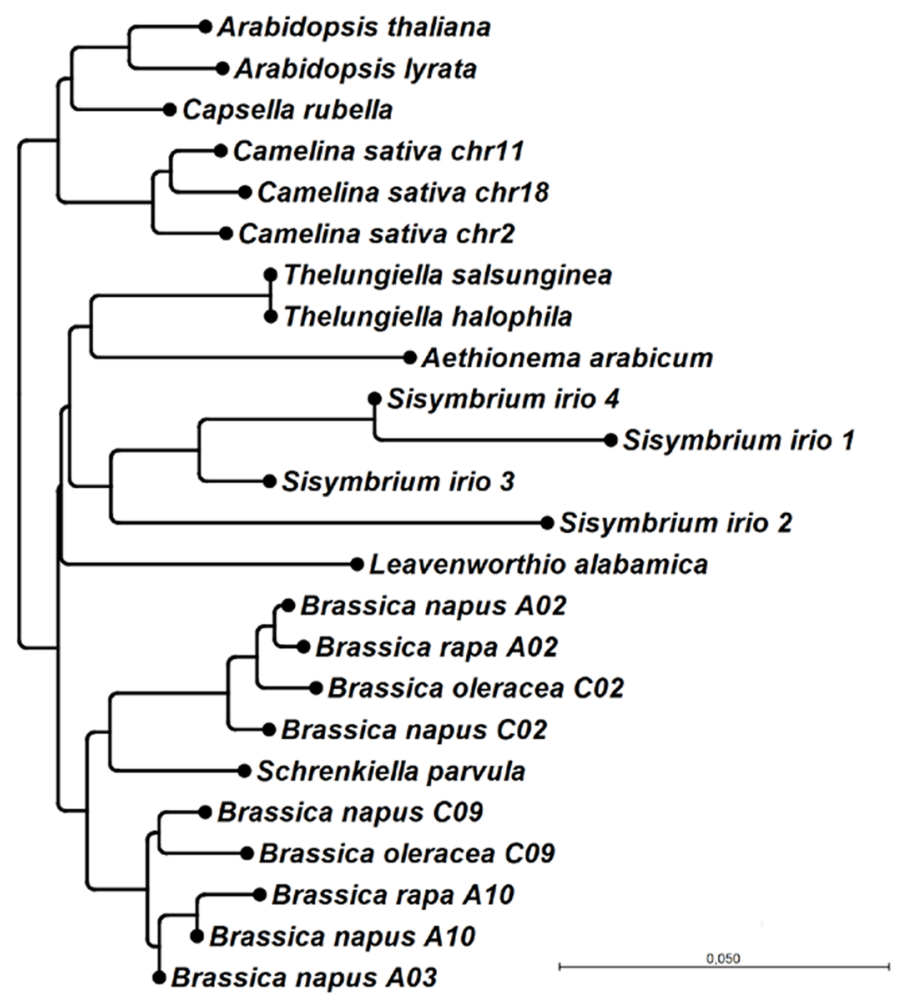

(b)

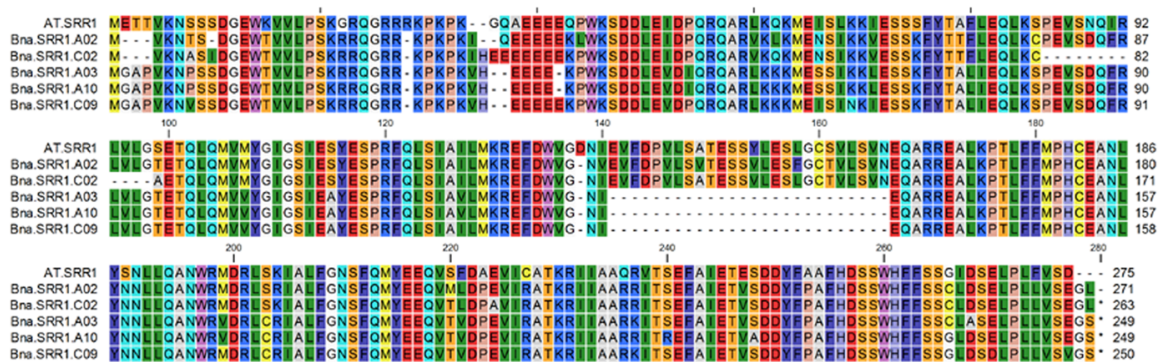

Fig. 1 a Neighbor-joining tree for predicted protein sequences of SRR1 copies in 13 different species of the Brassicaceae. Genomic sequences were extracted from BRAD. Sequence alignment was performed using CLUSTAL multiple sequence alignment by MUSCLE with Default parameters. Based on this alignment, neighbor joining tree using bootstrap analysis (100 replicates) was constructed using CLCSequenceViewer, version 8.0. b Full length alignment of the predicted amino acid sequences of At.SRR1 with the 5 Bna.SRR1 copies

Sequence comparisons indicate that the Bna.SRR1.A03 copy arose from a duplication of the Bna.SRR1.A10 copy (Fig. 1a).

Gene sequence analysis shows that the five Bna.SRR1 copies can be divided into two groups, based on their predicted amino acid sequence. The first group, consisting of the AO2 and CO2 gene copies, is more similar to the AtSRR1 protein although several amino acid changes have occurred (Fig. 1b). The second group, consisting of the A03, A10 and C09 gene copies, all have a 21 amino acid deletion in their protein sequences, compared to the
AtSRR1 protein and the $\mathrm{A} 02$ and $\mathrm{C} 02$ proteins (similarity to AtSRR1: A02: $83.6 \%$ and C02: $80.7 \%$ conservation vs A03: $73.4 \%, \mathrm{~A} 10: 73.8 \%$ and $\mathrm{C} 09$ 74.9\% conservation). Only one copy in B. rapa and B.oleracea and two copies in S. irio showed similar deletions in this region. A 13 amino acid deletion is also found in the $\mathrm{C} 02$ protein, which is unique for this homolog (Fig. 1b).

Not all Bna.SRR1 copies are expressed

By sampling the Manitoba winter type accession, requiring an extended period of cold to be able to flower, and 
the Korall spring type accession, which does not, potential seasonal differences in expression were examined. For 10 week old plants, emerging leaves, developed leaves and petioles were sampled and expression levels of the different copies were tested in the sampled tissues with RT-qPCR using copy-specific primers. This revealed that only three of the five gene copies were expressed at detectable levels, namely the $\mathrm{AO2}, \mathrm{CO} 2$ and the $\mathrm{CO} 9$ gene copies (Fig. 2). Of these, the C09 copy was expressed at higher levels compared to the other gene copies, accumulating to about two times the levels of the A02 copy in all tested tissues in the Manitoba winter type and to an even higher ratio in the Korall spring type (Fig. 2). The CO2 copy was expressed at lower levels than both the A02 and C09. In emerging leaves, all expressed gene copies were expressed at higher levels in the winter type Manitoba, compared to the spring type Korall (Fig. 2a). In developed leaves, expression levels were more similar between the accessions and the C09 copy was expressed at a slightly higher, but not significantly, level in the spring type Korall compared to the winter type Manitoba (Fig. 2b). In petioles, expression of the AO2 and $\mathrm{CO} 2$ copies was only detectable in the Manitoba winter type while the C09 copy was expressed at high levels in both Korall and Manitoba (Fig. 2c). Thus, there is a much more prominent difference in expression level between accessions in emerging leaves compared to developed leaves. This may suggest that the Bna.SRR1 genes have an important regulatory role at an earlier stage of development in the Manitoba winter accession compared to the Korall spring accession. To examine whether these findings were accession-specific or dependent on the winter type vernalization requirement, nine additional winter and spring accessions of the ASSYST collection $[42,43]$, were sampled for emerging leaf material and the expression of Bna.SRR1 A02, CO2 and $\mathrm{CO} 9$ was examined. Five accessions were classified as early flowering and four as late flowering of the winter types, while four accessions were early flowering and five late flowering of the examined spring types. Analysis of these accessions revealed a large variation in expression of the A02 gene copy between the accessions (Fig. 3a). Interestingly, the late-flowering spring lines had a statistically significant $(p>0.01$, two-factorial ANOVA) higher expression of the A02 copy compared to the early-flowering spring lines.

The $\mathrm{CO} 2$ gene copy was expressed differently between accessions, expression levels were generally higher in the winter accessions, but in several accessions, no expression at all was detected (Fig. 3b).

Expression of the $\mathrm{C} 09$ copy was more stable between the different accessions and comparable to what was observed in the Korall and Manitoba accessions, suggesting presence of the $\mathrm{CO}$ gene product is important in both winter and spring types (Fig. 3c). Additionally, to examine Bna.SRR1 expression in other tissues, roots, stems and flowers were sampled from the spring accession Ability as well as roots and stems from the winter accession Zephir. Expression of Bna.SRR1 was subsequently tested. No Bna.SRR1 gene copy could be detected in roots, while expression of A02, C02 and $\mathrm{CO} 9$ was detected in stems (Additional file 1: Figure S1). Here, the $A 02$ copy was expressed at higher levels than the $\mathrm{CO} 2$ and $\mathrm{CO} 9$ copies in the winter accession, while the C09 copy had a similar level of expression in both accessions in stems and in flowers in the spring accession. The copy on $\mathrm{C} 02$ was expressed at similar levels to $\mathrm{C} 09$ in stems in both accessions, but not detectable in flowers. In conclusion, the A02 and C09 copies were detected in stem and flower tissue, while the CO2 copy was only detected in stems, suggesting possible tissue-specific subfunctionalization between the gene copies.

\section{Bna.SRR1 gene copies show different ability to rescue early flowering in srr1-1}

To examine whether the Bna.SRR1 gene copies may have a similar function in flowering as the Arabidopsis
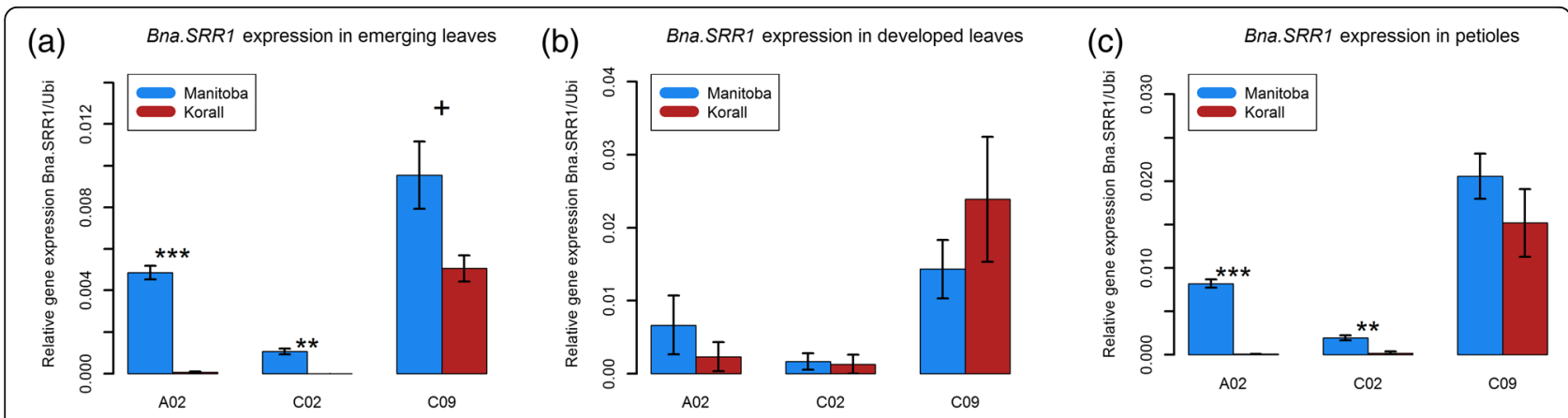

Fig. 2 Relative gene expression of the three expressed Bna.SRR1 copies in emerging and developed leaves and petioles in the accessions Manitoba (wintertype) and Korall (spring-type) without vernalization from rosette material with approximately 5 developed leaves. (a) Emerging leaves, (b) developed leaves, (c) petioles. The values were calculated from RT-qPCR using the $\Delta \mathrm{Ct}$ method and represent mean of 3 biological replicates. Error bars show standard error of mean. Asterisks show the level of significance based on the Student's t-test $\left({ }^{*} p\right.$-value $<0.05,{ }^{* *} p$-value $<0.01,{ }^{* * *} p$-value $<0.001$ ) 
(a) Bna.SRR1.A02 expression

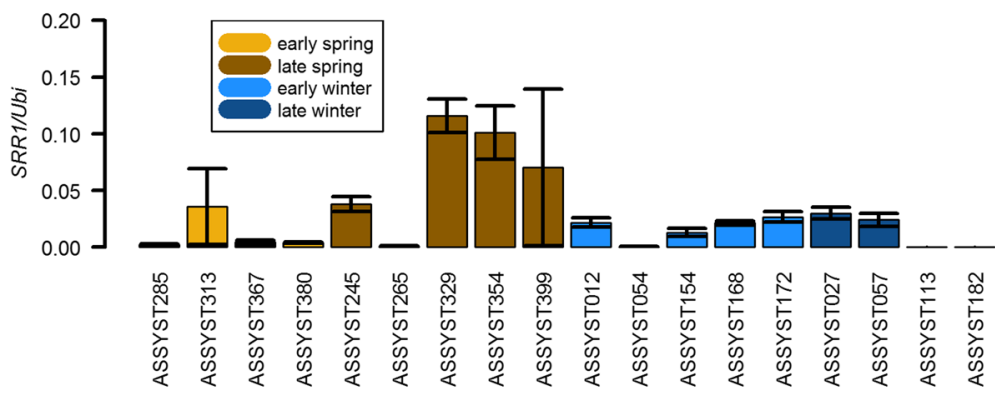

(b) Bna.SRR1.C02 expression

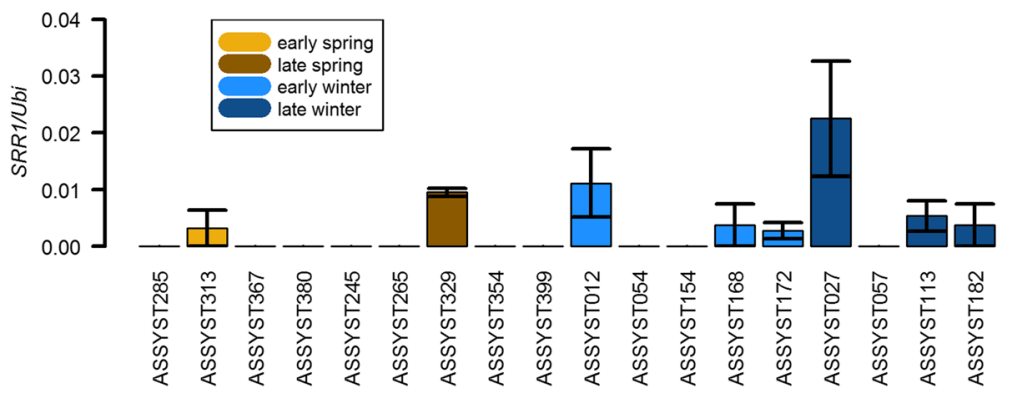

(c) Bna.SRR1.C09 expression

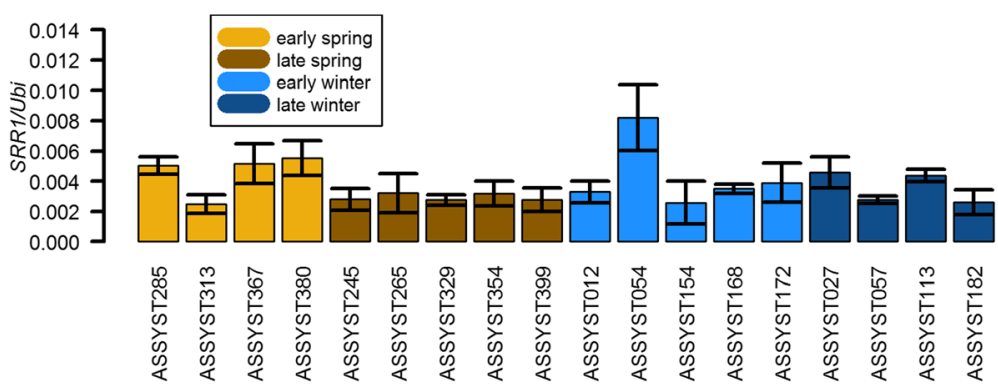

Fig. 3 Relative gene expression of Bna.SRR1 gene copies in early- and late-flowering spring and winter accessions from the ASSYST collection. (a) Bna.SRR1 A02, (b) Bna.SRR1 C02, (c) Bna.SRR1 C09. The values were calculated from RT-qPCR using the $\Delta C$ t method and represent mean of 3 biological replicates. Error bars show standard error of mean

SRR1 gene, the three gene copies shown to be expressed in B. napus (A02, CO2 and C09) were introduced into srr1-1 mutant plants. About $1500 \mathrm{bp}$ of the promoter region and the genes including the $3^{\prime}$ untranslated region were amplified from genomic $B$. napus DNA using PCR and introduced into the HPT1 binary vector [44]. Subsequently, srr1-1 mutant plants were transformed with these vectors to introduce the Bna.SRR1 copies into Arabidopsis. The transformed plant lines were tested for their flowering phenotype under SDs, where srr1-1 mutants are known to have a strong early flowering phenotype [1]. Flowering time of the transformed plant lines was then measured. The plants transformed with the AO2 gene copy as well as the CO2 copy flowered similar to Col-7 wt plants, thus fully complementing the early flowering phenotype of $\operatorname{srr} 1-1$ (Fig. 4a, b). In comparison, the plants transformed with the $\mathrm{CO}$ copy flowered with the same leaf numbers as the srr1-1 mutants (Fig. 4c). This suggests that the differences in CO9 compared to the other homologs may be critical for the proteins' ability to repress flowering in Arabidopsis. In contrast, the deletion in $\mathrm{CO} 2$ has no relevance for the function of the protein in regulation of flowering.

To examine how the difference in amino acid composition in C09 may alter the protein, the predicted protein structure of the different SRR1 copies was generated using the PredictProtein resource [45]. This showed that the SRR1 homologs are predicted to have a very similar structure (Additional file 2: Figure S2). The major difference in C09 compared to the other copies is that one $\alpha$ - 
(a)

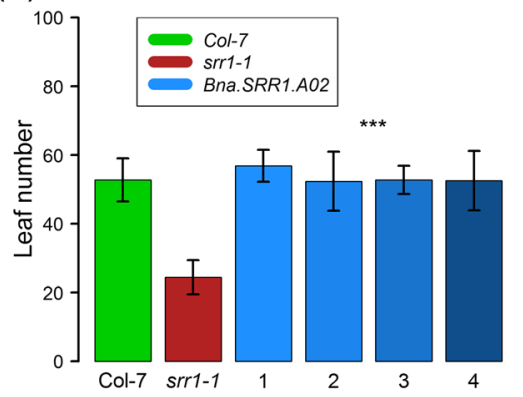

(b)

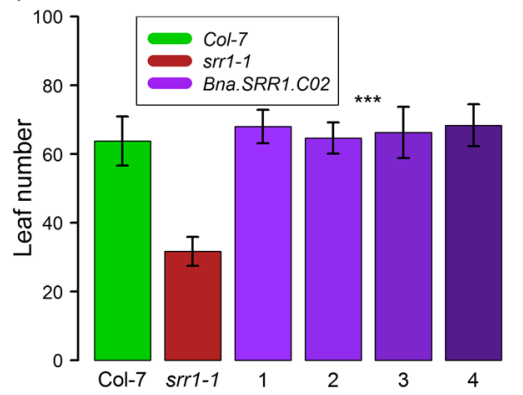

(c)

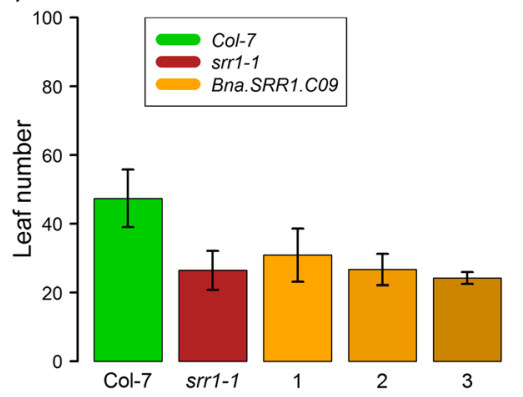

Fig. 4 Flowering time of srr $1-1$ plants transformed with Bna.SRR1 gene copies. Plants were grown in SD conditions (16 h light: $8 \mathrm{~h}$ dark, $\left.20^{\circ} \mathrm{C}\right)$. (a) Bna.SRR1 A02-transformed lines, (b) Bna-SRR1 C02-transformed lines, (c) Bna.SRR1 C09-transformed lines. Leaves of at least 15 plants per line were counted at flowering. Error bars show standard deviation. Asterisks show the level of significance based on the Student's t-test compared to not transformed srr1-1 plants ( ${ }^{*} p$-value $<0.05,{ }^{* *} p$-value $<0.01,{ }^{* * *} p$-value $\left.<0.001\right)$

helix, predicted to be mainly exposed, is missing through the deletion. The prediction does not suggest that the deletion renders the protein unstable.

\section{Expression of Bna.SRR1 gene copies in Arabidopsis}

As expression levels of the different Bna.SRR1 gene copies differed strongly in $B$. napus, the level of expression of Bna.SRR1.A02, which could complement flowering in Arabidopsis, and C09, which could not, was tested in the Arabidopsis lines transformed with the respective gene copies.

RT-qPCR analysis showed that in comparison to the endogenous SRR1 gene copy, both Bna.SRR1 genes introduced into the $\operatorname{srr} 1-1$ background were expressed at lower levels (Fig. 5). For the AO2 copy, these low levels of expression were obviously sufficient to complement the flowering phenotype. The C09 copy was also expressed at lower levels than AtSRR1, but higher than $A 02$ in the tested lines, reaching ca $30 \%$ of expression levels of AtSRR1. The level of expression of the AO2 copy does not appear to be critical for the function of SRR1, as low amounts of transcript are sufficient to fulfill its role in flowering time control. A comparison of the promoter structure between the SRR1 gene copies using the MEME suite [46] revealed two enriched motifs common in all the gene promoters, although their distribution is somewhat different between the genes (Additional file 3: Fig. S3). The motifs, a SORLIP motif and an ARF motif, have been described to be involved in light-regulated gene expression and as an auxin response factor binding site, respectively $[47,48]$. They were located close to the start of the coding sequence in

\section{SRR1 expression}

\section{SRR1 expression}
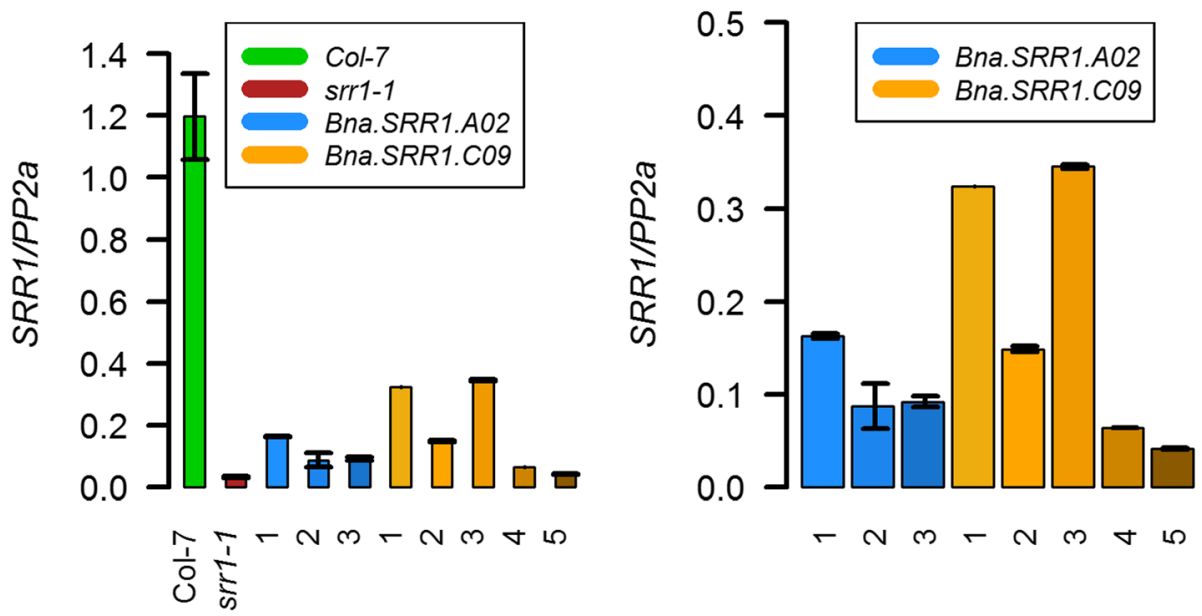

Fig. 5 Expression of SRR1 in Col-7 wt and srr1-1 lines transformed with the Bna.SRR1 gene copies. Left panel: Expression in comparison with Col-7 wt of three independent transformed lines transformed with Bna.SRR1 A02 and Bna.SRR1 C09, respectively. Right panel: magnified comparison of expression between the Bna.SRR1 A02-transformed and Bna.SRR1 C09-transformed lines. Error bars show standard error of mean 
AtSRR1, while they were located further upstream in the Bna.SRR1 gene promoters. Although the factors regulating SRR1 expression are unknown, this may indicate that the efficiency of transcriptional activation of the Bna.SRR1 genes in Arabidopsis is different, which could explain the reduced expression levels of the Bna.SRR1 copies compared to endogenous AtSRR1.

\section{Expression of SRR1 targets in Bna.SRR1 transformed lines}

SRR1 acts in several pathways regulating flowering by promoting the expression of $F T$ repressors [1]. To examine how the Bna.SRR1 copies affected known targets of AtSRR1 in regulation of gene expression, their transcript levels in plants carrying the AtSRR1-complementing A02 and the non-complementing C09 gene copies were measured.

To confirm that the complemented phenotype in BnaSRR1.A02 lines was due to restoration of the FT expression pattern, a time series was sampled in 3-h intervals over 24-h in SD conditions and analyzed using RT-qPCR. This revealed that in A02-transformed lines, $F T$ was expressed at very low wt-like levels, while elevated expression was observed in C09transformed lines, notably at the for flowering induction critical time point ZT9, as well as in srr1-1 mutants (Fig. 6a, Additional file 4: Fig. S4). Furthermore, analysis of $C D F 1$ expression, a known repressor of $F T$ and a target of SRR1 showed that CDF1, with an expected peak of expression in the morning, was expressed as in Col-7 in the lines transformed with the $A 02$ gene copy. Meanwhile, $C D F 1$ was expressed at reduced levels in the morning and expression peaked earlier in the C09-transformed lines (Fig. 6b). This was similar to the expression pattern observed in srr1-1 mutants and thus CO9 had no complementing effect on CDF1 expression.

TEM1 and TEM2 transcription factors are other known targets of SRR1, which are involved in regulation of flowering through the GA biosynthesis pathway [49]. Due to their redundancy and similar expression, TEM1 was examined to determine whether the Bna.SRR1 copies could affect their expression. Interestingly, whereas srr1-1 showed reduced expression, as seen previously [1], both the A02 and C09-transformed lines showed an expression pattern similar or even slightly enhanced compared to Col-7 wt, suggesting complementation of TEM1 expression (Fig. 6c). TEM1 is known to repress the gibberellic acid biosynthesis gene GIBBERELLIN 3-OXIDASE 1 (GA3OX1) [49]. To confirm the rescued expression of TEM1 in A02 and C09-transformed lines, GA3OX1 expression was measured. Transcript levels were elevated in srr1-1 compared to wt, congruent with previous observations [1]. In the C09-transformed lines no significant difference was seen while expression in A02-transformed lines was somewhat reduced (Additional file 5: Figure S5). The results support the elevated TEM1 levels in the transformed lines. In conclusion, this suggests that the function of SRR1 in transcriptional regulation is fully rescued by the $A 02$ gene copy for all tested genes, while the $C 09$ gene copy can only complement TEM1 expression, which is not enough to rescue the early flowering phenotype of srr1-1.

\section{Discussion}

Our data show that SRR1 is highly conserved in Brassicaceae, suggesting an important function in growth and development within this family (Fig. 1a). However, its unique protein structure has made the prediction of key regions of the protein or a molecular mode of action difficult. Interestingly, we found that the crop species Brassica napus carries in total five homologs with differences in gene and protein structure between them, including a larger deletion in the A03, A10 and C09 proteins, compared to the $\mathrm{A} 02$ and $\mathrm{C} 02$ proteins and AtSRR1 (Fig. 1b). As this could suggest subfunctionalization between the different gene copies, we tested their level of expression in $B$. napus followed by a functional analysis of the expressed copies in Arabidopsis srr1-1 background, where AtSRR1 is not expressed.
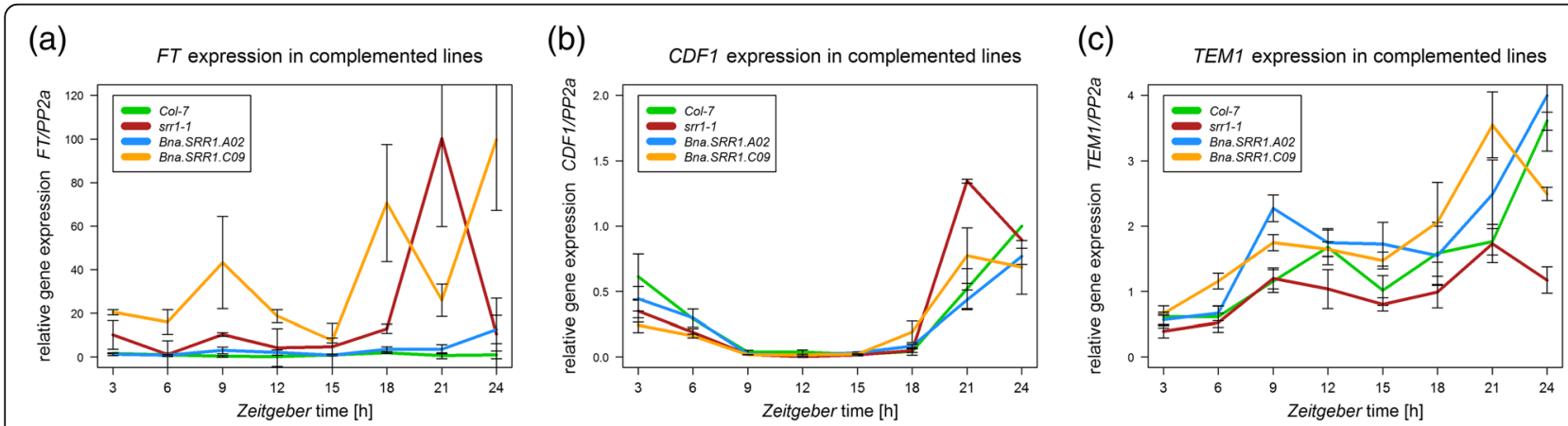

Fig. 6 Relative expression of known SRR1 targets in lines transformed with Bna.SRR1 A02 and Bna.SRR1.C09. (a) FT, (b) CDF1, (c) TEM1. The values represent the average expression of three independently transformed lines. Error bars show standard error of mean 


\section{Differential gene expression suggests} subfunctionalization

Initial gene expression analysis in the B. napus accessions Manitoba (winter type) and Korall (spring type) showed that only three of the five gene copies were expressed, A02, C02 and C09 (Fig. 2). Expression of the same copies was also detected in stems, while in flowers only AO2 and C09 was detected (Additional file 1: Figure S1). In roots, no Bna.SRR1 copy was detected.

Differential expression of $B$. napus flowering gene copies has been shown in several reports [25, 50-52]. Here, the $\mathrm{CO}$ copy is consistently expressed in all tested accessions and tissues, while the $A 02$ and $\mathrm{C} 02$ copies are expressed at different levels depending on accession in emerging leaves (Fig. 3).

In the winter type Manitoba and the spring type Korall, comparison of expression between developed and emerging leaves showed that differences in expression between accessions was lower in comparison to emerging leaves (Fig. 2a, b), suggesting that the $\mathrm{AO2}$ and $\mathrm{CO} 2$ gene copies may have a repressive role on flowering at early stages of development, when highly expressed. This may suggest that they have a comparable role as SRR1 in Arabidopsis, in suppressing flowering until the conditions are more favorable. Interestingly, a similar pattern has been observed for the important flowering time regulator, $F L C$, where three out of nine copies were differentially expressed between winter and spring material (Quezada et al., submitted). One copy of Bna.FLC was never expressed [53], similar to what we found for Bna.SRR1.A03 and Bna.SRR1.A10, indicating pseudogenization.

Thus, in Manitoba and Korall, the larger difference in Bna.SRR1 expression in emerging leaves compared to developed leaves between the Korall spring and the Manitoba winter type may suggest that high expression early in the developmental cycle in the winter type is desirable to prevent premature flowering. This could account for a mechanism measuring the ratio of developing to differentiated leaves, allowing flowering only after a certain leaf mass has been reached. Developing leaves could likewise send a "stop" signal, which is only overridden if enough differentiated tissue has developed.

However, in the extended analysis of emerging leaves of several other accessions, A02 expression displayed a large variation suggesting that such a mechanism may be accession-dependent. A02 was particularly highly expressed in several late-flowering spring accessions, suggesting it may have a role in delaying flowering in these accessions (Fig. 3a). This function may be overruled by $F L C$ in winter accessions with a vernalization requirement.

Expression of $\mathrm{CO} 2$ also varied between accessions, suggesting a possible accession-specific function, while expression of $\mathrm{CO}$ was much more stable between accessions in the extended analysis (Fig. 3b, c). In comparison, presence of the $\mathrm{CO} 9$ gene product seems to be of general importance in $B$ napus and thus have the different gene copies subfunctionalized to perform specific roles in this species.

\section{Complementation reveals potential key protein domain of SRR1}

Flowering time experiments with the three gene copies being expressed in B. napus showed that only the $A 02$ and $\mathrm{CO} 2$ gene copies can complement the early flowering phenotype of $\operatorname{srr} 1-1$ while the C09 copy cannot (Fig. 4). This suggests that the differences in C09 may be critical for the function of the SRR1 protein in Arabidopsis, at least in regard to its role in regulating flowering. The most obvious candidate region to be critical for correct function is the 21 amino acid deletion in C09. In comparison, although the $\mathrm{C} 02$ protein product also carries a deletion in another part of the protein, it could still complement the loss of AtSRR1 in srr1-1 plants (Fig. 1b, Fig. 4). As the SRR1 protein sequence does not contain any known regulatory elements, this is an important finding, indicating that this region of the protein may be critical for proper function. This deletion is a highly conserved SRR1-unique sequence in Brassicacea and this specific deletion only occurs in B. napus. Taking into account that the AO2 and CO2 copies are the same ones expressed at much lower levels in the spring type compared to the winter type, this further indicates that their expression may be necessary to prevent undesirable premature flowering in the winter type, acting as a repressive signal in months preceding the cold season.

The dysfunction of the C09 gene copy in Arabidopsis could be either due to an important function-specific binding region of the protein being excluded through the altered protein sequence, or due to direct degradation of the $\mathrm{C} 09$ protein product. However, the performed protein structure prediction suggests that $\mathrm{C} 09$ still has a similar structure to the other SRR1 copies, and only one predicted helix structure is missing (Additional file 2: Figure S2). Considering the experimental results, this deletion may be important for interactions or protein modifications necessary for the regulation of flowering time. Further biochemical studies are however necessary to confirm that the region deleted in $\mathrm{C} 09$ is the determining factor.

Considering that SRR1 in Arabidopsis is also involved in circadian regulation and light signaling [37], it is possible that the AO2 and $C 09$ copy may have a specialized functions in $B$. napus, through subfunctionalization. Being that the $C 09$ gene copy is unique for B. napus may also suggest a species-specific specialization. Its exact function requires more detailed analyses in B. napus. 
The expression analysis in lines complemented with $B$. napus gene copies in Arabidopsis shows that the expression levels of the introduced genes were much lower than the endogenous SRR1 in Col-7 wt plants (Fig. 5). This was, however, sufficient for $A 02$ to be able to complement the srr1-1 early flowering phenotype, suggesting that low SRR1 expression levels are enough for proper function. Expression of the C09 copy was lower than endogenous SRR1, but higher than Bna.SRR1.A02. Thus, considering that expression of the $A 02$ lines was sufficient to complement the flowering phenotype of $\operatorname{srr} 1-1$, it is unlikely that the level of C09 expression is a major factor in the inability of the $\mathrm{CO} 9$ gene copy to do the same (Fig. 5).

\section{CDF1 is key to regulate flowering through SRR1}

Analysis of known targets of SRR1 showed that the A02 gene copy was able to replace AtSRR1 function in regard to its role in regulating expression of flowering time regulators, including the key florigen $F T$, the important $F T$ repressor, $C D F 1$, and TEM1 (Fig. 6). In contrast, the C09 copy was unable to rescue SRR1 function, as the C09-transformed lines showed srr1-1-like expression patterns of CDF1 and FT. Conversely, TEM1 expression levels were rescued to WT levels by C09, but this seems to have a limited effect on flowering, as C09-transformed plants flowered like srr1-1 mutants. In conclusion, the data suggests that the key target for floral repression by SRR1 is $C D F 1$, where an altered expression is observed in $\operatorname{srr} 1-1$, as well as in C09-transformed lines (Fig. 6a). TEM1 appears to be rescued by both the A02 and C09 gene copies (Fig. 6c), although this is not enough to rescue the early flowering phenotype in C09-transformed lines. This indicates that the differences in C09, most notably the deleted region, may be necessary for SRR1 control of CDF1 expression.

Our data suggests that these gene copies may have a similar molecular mode of action in B. napus as in Arabidopsis and may be able to influence expression of $B$. napus homologs to other known flowering time components, which have been shown to be also present in $B$. napus [33]. Furthermore, the consistent expression levels of the C09 copy compared to the variation in A02 expression may suggest that the gene copies have subfunctionalized to acquire specific roles in B. napus that may or may not be related to the regulation of flowering. This information may help to map the signaling network controlling flowering time in B. napus, enabling the identification of key factors in breeding.

\section{Conclusions}

We have shown that SRR1, an important Arabidopsis flowering time regulator, has several homologs in Brassica napus. Their expression patterns varied and major alterations in amino acid composition were found. The differences in expression between winter and spring type accessions suggest their expression may be of importance to flowering ability.

Only two of three expressed copies could complement the early flowering srr 1-1 mutant phenotype, showing cross-species functionality. The C09 copy, with a 21 amino acid deletion compared to A02, C02, and AtSRR1, failed to complement the early flowering phenotype. $\mathrm{CO} 9$ is, however, consistently expressed in B. napus, suggesting strong subfunctionalization between the gene copies. The presented data may be used in the future for further characterization of the flowering time pathway in $B$. napus and highlights the possibility that the $B$. napus gene copies may have taken on specific functions throughout evolution.

\section{Methods}

\section{Sequence analysis}

Whole genome sequences for $A$. thaliana, A. lyrata, $B$. napus, B. rapa, B.oleracea, Camelina sativa, Capsella rubella, Thelungiella salsunginea, Thelungiella halophila, Aethionema arabicum, Leavenworthia alabamicum, Schrenkiella parvula and Sisymbrium irio were retrieved from http://brassicadb.org/brad/ftpTrans.php. The five known copies of B. napus were then used for a BLAST search against each of the genomes. Bna.SRR1 copies were then selected using a cut-off value of $10^{-50}$ for Brassica and Arabidopsis, while using a cutoff of $10^{-20}$ for the remaining species. Fragments shorter than $200 \mathrm{bp}$ were excluded. To avoid missing gene information, $100 \mathrm{bp}$ were added to the start and stop of each BLAST position. For all species except the Brassica species and Arabidopsis thaliana, peptide sequences were predicted using GENSCAN (http://genes.mit.edu/GENSCAN.html) with "Arabidopsis" as organism. For Brassica and A. thaliana, we used the peptide sequence information from the respective peptide prediction published within their reference genomes.

\section{Plant material and growth conditions Arabidopsis thaliana}

The T-DNA mutant srr1-1 in the Col-7 background has been described $[1,37]$. All seeds were stratified for $3 \mathrm{~d}$ at $4{ }^{\circ} \mathrm{C}$ before putting on soil. Seeds grown on plates were surface sterilized and stratified for $3 \mathrm{~d}$ at $4{ }^{\circ} \mathrm{C}$ before plating on agar-solidified half-strength MS (Murashige \& Skoog) medium (Duchefa) supplemented with $0.5 \%$ sucrose and 0.5 g MES. Plants were grown in Percival incubators AR66L3 (CLF Laboratories) in $100 \mu \mathrm{mol} \mathrm{m}^{-2} \mathrm{~s}^{-1}$ light intensity, with the light-dark and temperature conditions as indicated. 


\section{Brassica napus}

A winter accession (Manitoba) and a spring accessions (Korall) of oilseed rape were sown in $7 \times 7 \mathrm{~cm}$ pots in 3 biological replicates and transplanted to $12 \times 12 \mathrm{~cm}$ pots 4 weeks after sowing. For the extended expression analysis, a diversity set consisting of 10 winter and 10 spring accessions was sown in quickplates in 3 biological replicates. Cultivation was performed in a greenhouse using a $16 \mathrm{~h} / 8 \mathrm{~h}$ day/night rhythm with $20^{\circ} \mathrm{C} / 17^{\circ} \mathrm{C}$. For Manitoba and Korall, we sampled petioles, developed and emerging leaves separately 10 weeks after sowing. For the diversity set, we selected 9 winter and 9 spring accessions for the youngest developed leaf 8 weeks after sowing. The other two accessions were grown 3 weeks further and we sampled stems, roots and flowers separately. Tissues were frozen in liquid nitrogen and stored at $-80^{\circ} \mathrm{C}$ until RNA extraction.

\section{Flowering time experiments}

Seeds were germinated as described above and grown on soil in a random fashion. Flowering time was determined by counting the rosette leaves when the bolt was $>0.5$ $\mathrm{cm}$ tall [54].

\section{Cloning}

Genomic DNA from Brassica napus was amplified using Phusion Proofreading polymerase (Thermo Fischer) and primers with specific restriction sites. The amplified DNA was separated on an agarose gel and extracted using a GeneJet gel extraction kit (Thermo Fischer) and then ligated into a pJET2.1 cloning vector using the CloneJet kit (Thermo Fischer). The insert was digested and separated on an agarose gel and then cloned into a pHPT1 binary vector [44], using T4 Ligase (Thermo Fischer). The resulting construct was transformed into Agrobacterium and then into Arabidopsis srr1-1 plants using the floral dip method.

\section{Transcript analysis}

\section{Arabidopsis material}

Total RNA was extracted using from plant material using Tri Reagent as previously described or using Universal RNA Purification Kit (Roboklon) following manufacturer's instructions.

For cDNA, $2 \mu \mathrm{g}$ of total RNA was DNAse-treated using RQ1 RNAse-free DNAse (Promega) and reverse transcribed using AMV Reverse Transcripase (Roboklon) according to manufacturer's instructions.

qPCR was performed with iTaq Sybr Green Supermix (Bio-Rad) according to manufacturer's instructions. The normalized expression level was determined using the $\triangle$ Ct method, with PP2a (At1g69960) as a reference gene as described [55]. The primer sequences can be found in Additional file 6: Table S1.

\section{Brassica napus material}

Total RNA was extracted using the NucleoSpin miRNA kit (Macherey-Nagel) following manufacturer's instructions. The eluted RNA was quantified using Qubit RNA Broad Range on a Qubit fluorimeter and stored at $80^{\circ} \mathrm{C}$ until use.

Primers were designed based on the Darmor-bzh reference genome, version 4.1 (Chalhoub et al. 2014). Specificity was confirmed by aligning the predicted cDNA with CLUSTAL multiple sequence alignment by MUSCLE (http://www.ebi.ac.uk/Tools/msa/muscle/, version 3.8). The primer sequences can be found in Additional file 6: Table S1.

cDNA synthesis was performed using the RevertAid cDNA synthesis kit (ThermoFisher) using $1 \mu \mathrm{g}$ of total RNA and Oligo-dT primers. The amount of cDNA was quantified using the Qubit DNA High Sensitivity kit on a Qubit fluorimeter. Quantitative Real-time PCR was performed on a Real-Time PCR System ViiA7 cycler (Applied Biosystems) in 384 well plates. The reaction mix containing specific primers, the template cDNA and FastStart Universal SYBR Green Master mix containing Rox (Roche) was pipetted by a robot (Biomek 4000, Beckman Coulter). As endogenous control, we used ubiquitin. The PCR program was as follows: initial denaturation $\left(94{ }^{\circ} \mathrm{C}\right.$ for $2 \mathrm{~min}$ ), amplification and quantification $\left(40\right.$ cycles, $95^{\circ} \mathrm{C}$ for $20 \mathrm{~s}, 60^{\circ} \mathrm{C}$ for $30 \mathrm{~s}, 72^{\circ} \mathrm{C}$ for $30 \mathrm{~s}$ ), and a final extension $\left(72^{\circ} \mathrm{C}\right.$ for $\left.5 \mathrm{~min}\right)$. At the end, a melting curve was recorded between 55 and $95^{\circ} \mathrm{C}$. PCR efficiency was measured using a pool of all samples in a dilution series of 6 points. All samples were measured in 3 technical replicates. The normalized expression level was determined using the $\Delta \mathrm{Ct}$ method.

\section{Additional files}

Additional file 1: Figure S1. Relative gene expression of Bna.SRR1 gene copies in different tissues of the Ability spring and Zephir winter accessions. The values were calculated from RT-qPCR using the $\Delta \mathrm{Ct}$ method and represent mean of 3 biological replicates. Error bars show standard error of mean. (TIF 10547 kb)

Additional file 2: Figure S2. Protein structure predictions based on the PredictProtein server. Red squares in the first row indicate predicted alpha-helices, blue squares indicate strands. Yellow boxes in the second row indicate buried regions while blue boxes indicate exposed regions. Grey boxes in the third row indicate disordered regions. The red dotted squares highlight a predicted helix missing in Bna.C09 compared to the other predicted SRR1 copies. (TIF $517 \mathrm{~kb}$ )

Additional file 3: Figure S3. AtSRR1 and BnSRR1 promoter alignment. Two enriched motifs were discovered using MEME. Sequences from A.thaliana and B.napus $1 \mathrm{~kb}$ upstream from the transcriptional start site were used with a minimal motif length of 6 and maximum of 10 (Bailey and Elkan, Proc Int Conf Intell Syst Mol Biol, 2:28-36,1994). Motifs were determined to be statistically significant with an E-value lower than 0.05 . SORLIP 2 binding site is associated with PhyA signaling, while ARF (Auxin Response Factor) binding sites are intrinsic for the auxin response. Enriched motifs are underlined and binding sites are highlighted in gray. (TIF 577 kb) 
Additional file 4: Figure S4. Expression of FT at zeitgeber time $9(9 \mathrm{~h}$

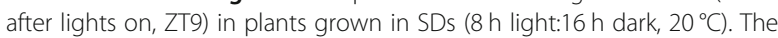
values represent biological replicates of three independently transformed lines. Error bars show standard error of mean. (TIF $5273 \mathrm{~kb}$ )

Additional file 5: Figure S5. Expression of TEM1 target GA3OX1 at zeitgeber time 8 ( $8 \mathrm{~h}$ after lights on, ZT8) in plants grown in SDs $(8 \mathrm{~h}$ light: $16 \mathrm{~h}$ dark, $20^{\circ} \mathrm{C}$ ). The values represent biological replicates of three independently transformed lines. Error bars show standard error of mean Asterisks show the level of significance based on the Student's t-test compared to Col-7 wt plants. (TIF $6591 \mathrm{~kb}$ )

Additional file 6: Primer sequences. (XLSX $10 \mathrm{~kb})$

\section{Abbreviations}

LD: Long day; RT-qPCR: Real Time Quantitative PCR; SD: Short day

\section{Acknowledgements}

This project was initiated in the DFG Priority Program SPP1530 on Flowering Time Control (STA 653/5).

\section{Consent to publication}

Not applicable.

\section{Authors'contributions}

MJ, SVS and DS designed the experiments. MJ, SVS, NW and PS performed experiments. MJ and SVS performed data analysis. MJ, SVS and DS wrote the manuscript with input from all authors. All authors have read and approved the final manuscript.

\section{Funding}

MJ is supported by the DFG grant JO 1252/2. SVS has been funded by grant SN14/14-2. The funding bodies had no role in the experimental design, data analysis, decision to publish, or preparation of the manuscript.

\section{Availability of data and materials}

All data generated or analysed during this study are included in this published article and its supplementary information files.

\section{Ethics approval and consent to participate}

Not applicable.

\section{Competing interests}

The authors declare that they have no competing interests.

\section{Author details}

${ }^{1}$ RNA Biology and Molecular Physiology, Faculty for Biology, Bielefeld University, Universitaetsstrasse 25, 33615 Bielefeld, Germany. ${ }^{2}$ Department of Plant Breeding, Justus Liebig University, IFZ Research Centre for Biosystems, Land Use and Nutrition, Heinrich-Buff-Ring 26-32, 35392 Giessen, Giessen, Germany.

Received: 20 December 2018 Accepted: 13 August 2019

Published online: 22 August 2019

\section{References}

1. Johansson M, Staiger D. SRR1 is essential to repress flowering in noninductive conditions in Arabidopsis thaliana. J Exp Bot. 2014;65(20):5811-22.

2. Johansson M, Staiger D. Time to flower: interplay between photoperiod and the circadian clock. J Exp Bot. 2015;66:719-30.

3. Jung C, Muller AE. Flowering time control and applications in plant breeding. Trends Plant Sci. 2009;14(10):563-73.

4. McClung CR, Lou P, Hermand V, Kim JA. The importance of ambient temperature to growth and the induction of flowering. Front Plant Sci. 2016;7:1266.

5. Hyun Y, Richter R, Coupland G. Competence to flower: age-controlled sensitivity to environmental cues. Plant Phys. 2017;173(1):36-46.

6. Song YH, Shim JS, Kinmonth-Schultz HA, Imaizumi T. Photoperiodic flowering: time measurement mechanisms in leaves. Annu Rev Plant Biol. 2015;66:441-64
7. An H, Roussot C, Suárez-López P, Corbesier L, Vincent C, Piñeiro M, et al. CONSTANS acts in the phloem to regulate a systemic signal that induces photoperiodic flowering of Arabidopsis. Development. 2004;131(15):3615-26.

8. Corbesier L, Vincent C, Jang SH, Fornara F, Fan QZ, Searle I, et al. FT protein movement contributes to long-distance signaling in floral induction of Arabidopsis. Science. 2007;316(5827):1030-3.

9. Jaeger KE, Wigge PA. FT protein acts as a long-range signal in Arabidopsis. Curr Biol. 2007;17(12):1050-4

10. Mathieu J, Warthmann N, Küttner F, Schmid M. Export of FT protein from phloem companion cells is sufficient for floral induction in Arabidopsis. Curr Biol. 2007;17(12):1055-60.

11. Suarez-Lopez P, Wheatley K, Robson F, Onouchi H, Valverde F, Coupland G. CONSTANS mediates between the circadian clock and the control of flowering in Arabidopsis. Nature. 2001;410(6832):1116-20.

12. Sawa M, Nusinow DA, Kay SA, Imaizumi T. FKF1 and GIGANTEA complex formation is required for day-length measurement in Arabidopsis. Science. 2007;318(5848):261-5.

13. Fornara F, Panigrahi KCS, Gissot L, Sauerbrunn N, Rühl M, Jarillo JA, et al. Arabidopsis DOF transcription factors act redundantly to reduce CONSTANS expression and are essential for a photoperiodic flowering response. Dev Cell. 2009:17(1):75-86.

14. Michaels SD, Amasino RM. FLOWERING LOCUS C encodes a novel MADS domain protein that acts as a repressor of flowering. Plant Cell. 1999;11(5):949-56.

15. Searle I, He Y, Turck F, Vincent C, Fornara F, Kröber S, et al. The transcription factor FLC confers a flowering response to vernalization by repressing meristem competence and systemic signaling in Arabidopsis. Genes Dev. 2006:20(7):898-912.

16. Chalhoub B, Denoeud F, Liu S, Parkin IA, Tang H, Wang X, et al. Plant genetics. Early allopolyploid evolution in the post-Neolithic Brassica napus oilseed genome. Science. 2014;345(6199):950-3.

17. Wang $X$, Wang H, Wang J, Sun R, Wu J, Liu S, et al. The genome of the mesopolyploid crop species Brassica rapa. Nat Genet. 2011:43(10):1035-9.

18. Liu S, Liu Y, Yang X, Tong C, Edwards D, Parkin IA, et al. The Brassica oleracea genome reveals the asymmetrical evolution of polyploid genomes. Nat Commun. 2014;5:3930

19. Parkin IA, Koh C, Tang H, Robinson SJ, Kagale S, Clarke WE, et al. Transcriptome and methylome profiling reveals relics of genome dominance in the mesopolyploid Brassica oleracea. Genome Biol. 2014; 15(6):R77.

20. Dassanayake M, Oh DH, Haas JS, Hernandez A, Hong H, Ali S, et al. The genome of the extremophile crucifer Thellungiella parvula. Nat Genet. 2011; 43(9):913-8.

21. Fletcher RS, Mullen JL, Heiliger A, McKay JK. QTL analysis of root morphology, flowering time, and yield reveals trade-offs in response to drought in Brassica napus. J Exp Bot. 2015;66(1):245-56.

22. Li X, Ramchiary N, Dhandapani V, Choi SR, Hur Y, Nou IS, et al. Quantitative trait loci mapping in Brassica rapa revealed the structural and functional conservation of genetic loci governing morphological and yield component traits in the a, B, and C subgenomes of Brassica species. DNA Res. 2013; 20(1):1-16.

23. Nelson MN, Rajasekaran $\mathrm{R}$, Smith $\mathrm{A}$, Chen $\mathrm{S}$, Beeck CP, Siddique KH, et al. Quantitative trait loci for thermal time to flowering and photoperiod responsiveness discovered in summer annual-type Brassica napus L. PLoS One. 2014;9(7):e102611.

24. Okazaki K, Sakamoto K, Kikuchi R, Saito A, Togashi E, Kuginuki Y, et al. Mapping and characterization of FLC homologs and QTL analysis of flowering time in Brassica oleracea. Theor Appl Genet. 2007;114(4):595-608.

25. Raman H, Raman R, Coombes N, Song J, Prangnell R, Bandaranayake C, et al. Genome-wide association analyses reveal complex genetic architecture underlying natural variation for flowering time in canola. Plant Cell Environ. 2016:39(6):1228-39.

26. Raman H, Raman R, Eckermann P, Coombes N, Manoli S, Zou X, et al. Genetic and physical mapping of flowering time loci in canola (Brassica napus L.). Theor Appl Genet. 2013;126(1):119-32.

27. Udall JA, Quijada PA, Lambert B, Osborn TC. Quantitative trait analysis of seed yield and other complex traits in hybrid spring rapeseed (Brassica napus L.): 2. Identification of alleles from unadapted germplasm. Theor Appl Genet. 2006:113(4):597-609.

28. Zhao J, Kulkarni V, Liu N, Del Carpio DP, Bucher J, Bonnema G. BrFLC2 (FLOWERING LOCUS C) as a candidate gene for a vernalization response QTL in Brassica rapa. J Exp Bot. 2010;61(6):1817-25. 
29. SchiessI S, Iniguez-Luy F, Qian W, Snowdon RJ. Diverse regulatory factors associate with flowering time and yield responses in winter-type Brassica napus. BMC Genomics. 2015;16:737.

30. Lagercrantz U, Putterill J, Coupland G, Lydiate D. Comparative mapping in Arabidopsis and Brassica, fine scale genome collinearity and congruence of genes controlling flowering time. Plant J. 1996;9(1):13-20.

31. Lysak MA, Koch MA, Pecinka A, Schubert I. Chromosome triplication found across the tribe Brassiceae. Genome Res. 2005;15(4):516-25.

32. Schiessl S, Huettel B, Kuehn D, Reinhardt R, Snowdon RJ. Targeted deep sequencing of flowering regulators in Brassica napus reveals extensive copy number variation. Sci Data. 2017:4:170013.

33. Schiessl S, Samans B, Huttel B, Reinhard R, Snowdon RJ. Capturing sequence variation among flowering-time regulatory gene homologs in the allopolyploid crop species Brassica napus. Front Plant Sci. 2014;5:404

34. Renny-Byfield S, Wendel JF. Doubling down on genomes: polyploidy and crop plants. Am J Bot. 2014;101(10):1711-25

35. Becker A, Theissen G. The major clades of MADS-box genes and their role in the development and evolution of flowering plants. Mol Phylogenet Evol. 2003;29(3):464-89.

36. Dreni L, Kater MM. MADS reloaded: evolution of the AGAMOUS subfamily genes. New Phytol. 2014;201(3):717-32.

37. Staiger D, Allenbach L, Salathia N, Fiechter V, Davis SJ, Millar AJ, et al. The Arabidopsis SRR1 gene mediates phyB signaling and is required for normal circadian clock function. Genes Dev. 2003;17(2):256-68.

38. Brachi B, Faure N, Horton M, Flahauw E, Vazquez A, Nordborg M, et al. Linkage and association mapping of Arabidopsis thaliana flowering time in nature. PLoS Genet. 2010;6(5):e1000940.

39. Fiechter V, Cameroni E, Cerutti L, Virgilio C, Barral Y, Fankhauser C. The evolutionary conserved BER1 gene is involved in microtubule stability in yeast. Curr Genet. 2008;53(2):107-15.

40. Xiao D, Zhao JJ, Hou XL, Basnet RK, Carpio DPD, Zhang NW, et al. The Brassica rapa FLC homologue FLC2 is a key regulator of flowering time, identified through transcriptional co-expression networks. J Exp Bot. 2013; 64(14):4503-16.

41. Schiessl S, Huettel B, Kuehn D, Reinhardt R, Snowdon R. Postpolyploidisation morphotype diversification associates with gene copy number variation. Sci Rep. 2017;7:41845.

42. Bus A, Korber N, Snowdon RJ, Stich B. Patterns of molecular variation in a species-wide germplasm set of Brassica napus. Theor Appl Genet. 2011; 123(8):1413-23.

43. Korber N, Wittkop B, Bus A, Friedt W, Snowdon RJ, Stich B. Seedling development in a Brassica napus diversity set and its relationship to agronomic performance. Theor Appl Genet. 2012;125(6):1275-87.

44. Schöning JC, Streitner C, Meyer IM, Gao Y, Staiger D. Reciprocal regulation of glycine-rich RNA-binding proteins via an interlocked feedback loop coupling alternative splicing to nonsense-mediated decay in Arabidopsis. Nucleic Acids Res. 2008:36(22):6977-87.

45. Yachdav G, Kloppmann E, Kajan L, Hecht M, Goldberg T, Hamp T, et al. PredictProtein--an open resource for online prediction of protein structural and functional features. Nucleic Acids Res. 2014;42:W337-43.

46. Bailey TL, Boden M, Buske FA, Frith M, Grant CE, Clementi L, et al. MEME suite: tools for motif discovery and searching. Nucleic Acids Res. 2009; 37(suppl_2):W202-W8.

47. Hudson ME, Quail PH. Identification of promoter motifs involved in the network of phytochrome A-regulated gene expression by combined analysis of genomic sequence and microarray data. Plant Physiol. 2003; 133(4):1605-16.

48. Ulmasov T, Hagen G, Guilfoyle TJ. Dimerization and DNA binding of auxin response factors. Plant J. 1999;19(3):309-19.

49. Osnato M, Castillejo C, Matías-Hernández L, Pelaz S. TEMPRANILLO genes link photoperiod and gibberellin pathways to control flowering in Arabidopsis. Nature Comm. 2012;3:808.

50. Tadege M, Sheldon CC, Helliwell CA, Stoutjesdijk P, Dennis ES, Peacock WJ. Control of flowering time by FLC orthologues in Brassica napus. Plant J. 2001;28(5):545-53.

51. Wang J, Hopkins CJ, Hou J, Zou X, Wang C, Long Y, et al. Promoter variation and transcript divergence in Brassicaceae lineages of FLOWERING LOCUS T. PLoS One. 2012;7(10):e47127.

52. Wang N, Qian W, Suppanz I, Wei L, Mao B, Long Y, et al. Flowering time variation in oilseed rape (Brassica napus $L$.) is associated with allelic variation in the FRIGIDA homologue BnaA.FRI.a. J Exp Bot. 2011;62(15):5641-58.
53. Zou X, Suppanz I, Raman H, Hou J, Wang J, Long Y, et al. Comparative analysis of FLC homologues in Brassicaceae provides insight into their role in the evolution of oilseed rape. PLoS One. 2012;7(9):e45751.

54. Steffen A, Fischer A, Staiger D. Methods Mol Biol. 2014;1158:285-95.

55. Streitner C, Hennig L, Korneli C, Staiger D. Global transcript profiling of transgenic plants constitutively overexpressing the RNA-binding protein AtGRP7. BMC Plant Biol. 2010;10(1):221.

\section{Publisher's Note}

Springer Nature remains neutral with regard to jurisdictional claims in published maps and institutional affiliations.
Ready to submit your research? Choose BMC and benefit from:

- fast, convenient online submission

- thorough peer review by experienced researchers in your field

- rapid publication on acceptance

- support for research data, including large and complex data types

- gold Open Access which fosters wider collaboration and increased citations

- maximum visibility for your research: over $100 \mathrm{M}$ website views per year

At $\mathrm{BMC}$, research is always in progress.

Learn more biomedcentral.com/submissions 